\title{
Estructura factorial del Teacher Interpersonal Self-Efficacy Scale en docentes dominicanos: ¿uno o tres factores?
}

\author{
Factorial structure of the Teacher Interpersonal Self-Efficacy Scale \\ for Dominican Teachers: one or three factors?
}

\author{
Dinorah García ${ }^{1}$, Consuelo Cerviño ${ }^{2}$, Irene Fernández ${ }^{3 *}$, José Manuel Tomás ${ }^{3}$ \\ 1 - Instituto de Investigación Superior Pedro Poveda, República Dominicana. \\ 2 - Departamento de Psicología Evolutiva y de la Educación, Facultad de Psicología, Universidad de Valencia, España. \\ 3 - Departamento de Metodología de las Ciencias del Comportamiento, Facultad de Psicología, Universidad de Valencia, España.
}

Introducción

Método

Resultados

Discusión y

conclusiones

Referencias

Recibido: 16/08/2017 Revisado: 20/09/2017 Aceptado: 05/10/2017

\section{Resumen}

La autoeficacia docente se define como la creencia específica acerca de la externalidad o internalidad del control de las actividades que realiza el profesor en su trabajo. El objetivo del presente estudio es contribuir a entender las propiedades psicométricas de la escala 'Teacher Interpersonal Self-Efficacy Scale' en una muestra de docentes de República Dominicana. Se pusieron a prueba los modelos factoriales propuestos en la literatura mediante análisis factorial confirmatorio: a) un único factor de $a u$ toeficacia; b) dos factores de autoeficacia percibida: en la gestión del aula y en la obtención de apoyo; y c) tres factores de autoeficacia percibida: en la gestión del aula, en la obtención de apoyo de los compañeros, y en la obtención de apoyo de los superiores. Los tres modelos mostraron un ajuste que varía entre bueno y excelente. Se retuvo el modelo de tres factores. Se han incluido las implicaciones y limitaciones derivadas de la investigación.

Palabras clave: autoeficacia docente, profesores dominicanos, estructura factorial

\begin{abstract}
Teacher self-efficacy is defined as the specific belief about the degree to which activities carried out by the subject are either externally or internally controlled. The aim of this research is to contribute to the understanding of the psychometric properties of the Teacher Interpersonal SelfEfficacy Scale in a sample of teachers of the Dominican Republic. All models documented in the literature were tested by means of confirmatory factor analysis: a) a single factor of self-efficacy; b) two factors of perceived self-efficacy: in classroom management and in eliciting support, and c) three factors of perceived self-efficacy: in classroom management, in eliciting support from colleagues, and in eliciting support from school principals. All three models presented a good-to-excellent fit. The three-factor model was retained. Implications and limitations derived from the research have been included.
\end{abstract}

Keywords: teacher self-efficacy, Dominican teachers, factorial structure

*Correspondencia a: I. Fernández. Avd. Blasco Ibáñez, 21, 46010, Valencia, España. ifermar@outlook.com

Cómo citar este artículo: García, D., Cerviño, C., Fernández, I., \& Tomás, J. M. (2017). Estructura factorial del Teacher Interpersonal Self-efficacy Scale en docentes dominicanos: ¿uno o tres factores? Revista Evaluar, 17(2), 154-164. Recuperado de https://revistas.unc.edu.ar/index.php/revaluar 


\section{Introducción}

El concepto de autoeficacia surge a partir de la teoría socio-cognitiva de Bandura y la teoría del aprendizaje social de Rotter (Chacón, 2006). Siguiendo la línea teórica de Bandura, la autoeficacia se relaciona con los juicios que cada sujeto se forma sobre su propia capacidad para realizar una actividad específica. Así, la conducta humana se ve regulada por la auto-influencia, que se compone principalmente de la creencia sobre la eficacia personal (Bandura, 2000). Lo anterior nos permite pensar que, a menos que uno mismo se crea capaz de producir el efecto deseado mediante una acción, tiene poco incentivo para actuar. De esta forma, la autoeficacia percibida posee un rol importante en el incentivo potencial de los resultados esperados. La autoeficacia se relaciona con la conducta a través de procesos cognitivos, motivacionales, afectivos y de selección (Prieto, 2007). Las creencias de autoeficacia pueden influir en los patrones de pensamiento y actuar como un incentivo o un inhibidor de la conducta. Además, la autoeficacia influye en la competencia, de manera que para que un individuo actúe de forma competente necesitará un equilibrio permanente entre su percepción de autoeficacia y los conocimientos y habilidades que requiere para realizar con éxito una acción específica (Velázquez, 2012). Así, la conducta se adquiere y regula mediante el sentimiento personal de autoeficacia (Gonzalo \& León, 1999).

Cuando aplicamos estos conceptos al espacio escolar, naturalmente surge el interés por la autoeficacia docente, que comenzó entendiéndose como el grado en el que el docente se siente capaz de incidir en el rendimiento de sus estudiantes (Bergman, McLaughlin, Bass, Pauly, \& Zellman, 1977). Sin embargo, más recientemente se ha conceptualizado la autoeficacia docente como la creencia específica acerca de la externalidad o in- ternalidad del control de las actividades que realiza el sujeto (Fernández, 2012). Según Bandura (1993), el sentimiento de autoeficacia de los docentes para motivar y promover el aprendizaje afecta los tipos de ambiente de aprendizaje que forman, y el éxito académico que sus estudiantes son capaces de alcanzar. Así, el sentimiento de autoeficacia influye directamente en las prácticas pedagógicas que los docentes llevan a cabo (Chacón, 2006). A su vez, la autoeficacia se ve alterada por una serie de elementos, de acuerdo con lo establecido por Prieto (2007), entre los cuales se encuentran: el nivel de rendimiento de los estudiantes, la edad de los estudiantes, el nivel de preparación del profesorado y el tamaño de la clase. La autoeficacia es directamente proporcional a los tres primeros elementos e indirectamente proporcional al cuarto elemento, esto es, al tamaño de la clase. Con todo, se puede concebir a los docentes con un sentimiento de autoeficacia alto como aquellos que se interesan por el aprendizaje de sus estudiantes, experimentan libertad para tomar decisiones y manifiestan satisfacción con su tarea.

Los instrumentos utilizados para medir la autoeficacia docente incluyen: Teacher Locus of Control (TLC; Rose \& Meadway, 1981), Responsibility for Student Achievement (RSA; Guskey, 1981), Webb Efficacy Scale (WES; Ashton, Olejnik, Crocker, \& McAuliffe, 1982), Teacher Efficacy Scale (TES; Gibson \& Dembo, 1984), Science Teaching Efficacy Belief Instrument (STEBI; Riggs \& Enochs, 1990), Ashton Vignettes (Ashton, Buhr, \& Crocker, 1984) y Teacher Self-Efficacy Scale (TSS; Bandura, 1990). De todos estos, el Teacher Efficacy Scale (Gibson \& Dembo, 1984) es uno de los instrumentos más utilizados según diversos autores (Henson, Kogan, \& Vacha-Haase, 2001; Brouwers, Tomic, \& Stijnen, 2002). En el desarrollo de esta escala, los autores asumieron que 
el constructo de autoeficacia comprendía tanto la autoeficacia docente general -la influencia que un profesor cree que los profesores en general tienen sobre los alumnos- como la personal -la influencia que un docente en particular cree poseer sobre sus alumnos. Sin embargo, la sensación de autoeficacia general resulta problemática puesto que, según lo argumentado por Bandura (1997), las creencias de autoeficacia suelen variar para cada individuo según las tareas específicas por las que se pregunte. Con el fin de solventar esta controversia, se desarrolló la escala 'Teacher Interpersonal Self-Efficacy Scale' (TISES; Brouwers \& Tomic, 2001).

La escala 'Teacher Interpersonal SelfEfficacy Scale' comprende tres actividades interpersonales de los docentes: la gestión del comportamiento del alumnado en clase, la obtención del apoyo docente y la obtención del apoyo de los superiores. Esta escala ha sido solamente validada en Holanda (Brouwers \& Tomic, 2001) y en España (García-Ros, Fuentes, \& Fernández, 2015). La escala original en holandés fue validada por Brouwers y Tomic (2001), empleando una muestra de calibración y otra de validación. Se pusieron a prueba un modelo nulo (base de comparación), un modelo de un factor, otro de dos factores correlacionados y finalmente uno de tres factores correlacionados. En ambas muestras el modelo de tres factores correlacionados, tras la depuración de un ítem, mostró el mejor ajuste, si bien este puede considerarse sólo marginalmente bueno. Por su parte, en la validación de la escala en alumnos españoles (García-Ros et al., 2015) se pusieron a prueba tres estructuras factoriales, la teórica - $\mathrm{o}$ a priori- de tres factores correlacionados, una estructura de dos factores correlacionados y la más parsimoniosa de un solo factor. El ajuste de los modelos no fue plenamente satisfactorio, aunque mediante la estimación de cinco covarianzas entre los errores se consiguió un ajuste satisfactorio del modelo de tres factores correlacionados.

El objetivo de esta investigación es contribuir a entender las propiedades psicométricas de la escala 'Teacher Interpersonal Self-Efficacy Scale', y en concreto la capacidad de generalización de esta a muestras de docentes de otras poblaciones, concretamente a docentes de República Dominicana. Los objetivos específicos son: (a) analizar la estructura factorial mediante técnicas confirmatorias, poniendo a prueba todos los modelos alternativos que se han empleado en la literatura para la escala y (b) estimar la consistencia interna de la escala. Todos estos análisis se realizarán en una muestra probabilística de docentes dominicanos.

\section{Método \\ Muestra}

La muestra consistió en un total de 633 docentes, de los cuales un $49.9 \%$ pertenecían al Distrito 5 de La Vega y el $50.1 \%$ restante al Distrito 3 de Santo Domingo, en la República Dominicana. El procedimiento de obtención de la muestra fue de carácter aleatorio por conglomerados. Los distritos educativos de Santo Domingo y La Vega fueron los conglomerados, se escogieron de entre estos los 3 y 5 al azar, y de ellos se extrajo la muestra de docentes. La muestra está compuesta por un $79.5 \%$ de mujeres y un $20.5 \%$ de hombres. En cuanto al estado civil, la muestra presenta un $63.7 \%$ de casados, un $16.6 \%$ de solteros y el resto, $19.7 \%$, en situaciones alternativas.

\section{Instrumentos}

Para el propósito de este estudio psicométrico, se tuvo en cuenta la escala Teacher Inter- 
personal Self-Efficacy Scale (Brouwers \& Tomic, 2001). Esta escala consta de 24 ítems, medidos mediante una escala Likert de cinco anclajes, desde 1 (Totalmente en desacuerdo) a 5 (Totalmente de acuerdo). El instrumento mide a priori tres dimensiones de autoeficacia percibida: gestión del aula, obtención de apoyo de los compañeros y obtención de apoyo de los superiores. La primera dimensión, autoeficacia percibida en la gestión del aula, está compuesta por los ítems 1, 4, 5, 8, $9,10,11,13,14,15,17,18,22$ y 24 . Un ítem característico de esta dimensión es, por ejemplo: $\mathrm{Si}$ un alumno interrumpe la clase, soy capaz de reconducirlo rápidamente. Estos ítems fueron desarrollados por Emmer y Hickman (1991) y posteriormente adaptados a la sub-escala por Brouwers y Tomic (2001). La segunda dimensión, autoeficacia percibida en la obtención de apoyo de los compañeros, incluye los ítems 3, 7, 20, 21 y 23. Un ítem propio de este factor es: Si me enfrento a un problema en el que mis compañeros me pueden ayudar, soy capaz de acercarme a ellos para ello. La tercera y última dimensión, autoeficacia percibida en la obtención de apoyo de los superiores, comprende los ítems 2, 6, 12, 16 y 19. Un ejemplo de estos ítems es: Cuando es necesario, soy capaz de sacar a colación problemas con los directivos. Estas dos dimensiones relacionadas con el apoyo fueron desarrolladas por Brouwers (2000).

Como criterios en una red nomológica se han considerado otros constructos medidos en la misma investigación, Loyola Generativity Scale (LGS; McAdams \& St Aubin, 1992) y Escala de Motivaciones Psicosociales (Fernández, 1987). La LGS tiene un primer factor que mide la expresión generativa y un segundo factor de carencia generativa. Los índices alfa de esta escala fueron .89 y .75 , respectivamente. Por su parte, la Escala de Motivaciones Psicosociales está formada por tres factores: motivación intrínseca, con alfa de
.839, motivación extrínseca, con alfa de .79, y motivación intrínseca para el desarrollo profesional, con alfa de 67.

\section{Análisis estadísticos}

En esta investigación se evaluaron los modelos puestos a prueba en el artículo original de Brouwers y Tomic (2001) mediante análisis factorial confirmatorio (AFC). El Modelo 1 consta de un único factor de autoeficacia. El Modelo 2 está compuesto por dos factores: autoeficacia percibida en la gestión del aula y autoeficacia percibida en la obtención de apoyo. Y el Modelo 3 cuenta con tres factores: autoeficacia percibida en la gestión del aula, autoeficacia percibida en la obtención de apoyo de los compañeros, y autoeficacia percibida en la obtención de apoyo de los superiores. Los modelos se muestran en la Figura 1. Se calcularon varios índices de ajuste, tal y como recomienda la literatura (Hu \& Bentler, 1999; Tanaka, 1993): el estadístico chi-cuadrado $\left(\chi^{2}\right)$, el índice de ajuste comparativo (CFI), y el error de aproximación de la media cuadrática (RMSEA). Generalmente se considera un buen ajuste con un CFI $>.90$ y RMSEA $<.08$, y excelente con CFI $>.95$ y RMSEA < .05 (Hu \& Bentler, 1999). El CFI mide la mejora en el ajuste frente a un modelo nulo, mientras que el RMSEA es una medida de la cuantía del error. Se empleó el método de estimación de los mínimos cuadrados ponderados y varianza corregida (WLSMV), puesto que los ítems fueron medidos como variables categóricas (ordinales) y la literatura recomienda este método cuando las variables son de dicha naturaleza (Finney \& DiStefano, 2006), además de no-normales.

Como medidas de fiabilidad de los factores se estimaron los valores tanto del alfa de Cronbach como del índice de confiabilidad compuesto 

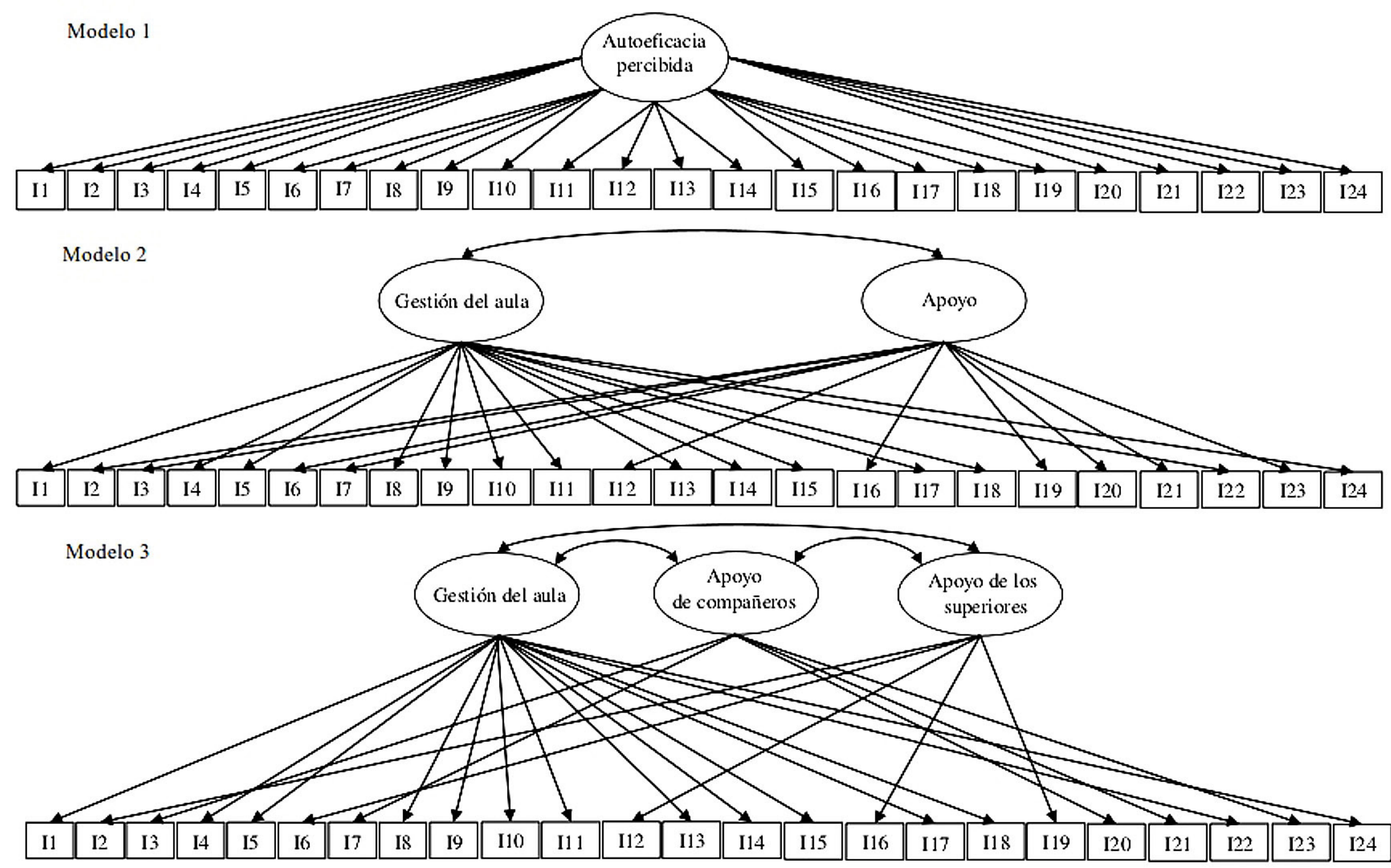

Figura 1

Modelos puestos a prueba en el estudio: a) un único factor de autoeficacia; b) dos factores: autoeficacia percibida en la gestión del aula y autoeficacia percibida en la obtención de apoyo; y c) tres factores: autoeficacia percibida en la gestión del aula, autoeficacia percibida en la obtención de apoyo de los compañeros, y autoeficacia percibida en la obtención de apoyo de los superiores. 
Tabla 1

Índices de ajuste para los modelos testados.

\begin{tabular}{lcccccc}
\hline Modelos & $\boldsymbol{\chi}^{2}$ & $\boldsymbol{g l}$ & $\boldsymbol{p}$ & CFI & RMSEA & CI 90\% \\
\hline Modelo 1 & 1754.361 & 252 & $<.001$ & .943 & .097 & $.093-.102$ \\
Modelo 2 & 1505.824 & 251 & $<.001$ & .953 & .089 & $.085-.094$ \\
Modelo 3 & 1469.382 & 249 & $<.001$ & .954 & .088 & $.084-.093$ \\
\hline
\end{tabular}

Tabla 2

Correlaciones entre los factores de autoeficacia y los criterios de su red nomológica.

\begin{tabular}{|c|c|c|c|c|c|}
\hline Autoeficacia & $\begin{array}{c}\text { Expresión } \\
\text { Generativa }\end{array}$ & $\begin{array}{c}\text { Carencia } \\
\text { Generativa }\end{array}$ & $\begin{array}{l}\text { Motivación } \\
\text { Intrínseca }\end{array}$ & $\begin{array}{l}\text { Motivación } \\
\text { Extrínseca }\end{array}$ & $\begin{array}{c}\text { Motivacion } \\
\text { Intrínseca para } \\
\text { Desarrollo } \\
\text { Profesional }\end{array}$ \\
\hline Autoeficacia General & $.609 * *$ & .066 & $.585 * *$ & -.069 & $.239 * *$ \\
\hline Gestión del Aula & $.612 * *$ & -.083 & $.590 * *$ & $-.109 *$ & $.241 * *$ \\
\hline Apoyo Compañeros & $.454 * *$ & $-.098 *$ & $.437 * *$ & $-.163 * *$ & .060 \\
\hline Apoyo Superiores & -.018 & $.297 * *$ & -.002 & $.150 * *$ & $.115 * *$ \\
\hline
\end{tabular}

Nota. ${ }^{* *} p<.01 ; * p<.05$

(CRI). Finalmente, se relacionaron los factores encontrados en la escala con una serie de criterios de su red nomológica, mediante coeficientes de correlación de Pearson. Los coeficientes alfa y las correlaciones de Pearson se calcularon en SPSS 21, mientras que los análisis factoriales confirmatorios y los CRI se calcularon en MPLUS 7 (Muthén \& Muthén, 1998-2012).

\section{Resultados}

Estructura factorial

Los índices de ajuste de los tres modelos probados se muestran en la Tabla 1. Los tres modelos presentan un ajuste de bueno a excelente, pero el que mejores índices de ajuste presenta es el Modelo 3. El modelo de dos factores tiene un ajuste también excelente, sin embargo, la correlación entre los dos factores es muy alta, de .92 $(p<$
.01), lo que indica una baja validez discriminante entre los dos factores. Por su parte las correlaciones entre los factores del Modelo 3 son también muy elevadas. Así, se observa que gestión del aula se correlaciona un .90 $(p<.001)$ con obtención de apoyo de los compañeros, un $.89(p<.01)$ con la obtención de apoyo de superiores, y obtención de apoyo de los compañeros y obtención de apoyo de superiores correlacionan $.93(p<.001)$.

\section{Consistencia interna}

Se calcularon el alfa de Cronbach y el CRI como estimadores de la consistencia interna de la escala. En el modelo de un solo factor, de autoeficacia docente general, el alfa fue de .91, mientras que en el modelo de tres factores los alfas fueron de .84 para gestión del aula, .786 para obtención de apoyo de los compañeros y .75 para obtención 
de apoyo de los superiores. En cuanto al CRI, se obtuvo un valor de .95 para el modelo de un solo factor, valores de .91 para gestión del aula, .886 para obtención de apoyo de los compañeros y .86 para obtención de apoyo de los superiores.

\section{Validez discriminante}

Los resultados de relacionar los factores de la escala TISES con una serie de criterios de su red nomológica (generatividad y motivación) se presentan en la Tabla 2. Se ofrecen tanto las correlaciones de la solución de un factor como la de tres factores (los dos modelos mejores para representar los datos). Puede verse cómo la autoeficacia, como factor general, correlaciona alto y según lo esperado con generatividad y motivación intrínseca. Al dividir los ítems en las tres dimensiones del modelo de tres factores, puede apreciarse cómo estas no se comportan igual, más bien exhiben un comportamiento claramente idiosincrásico del factor obtención de apoyo de los superiores.

\section{Discusión y conclusiones}

Los resultados factoriales obtenidos no resultan tan claros como sería deseable. Por un lado, el modelo de tres factores propuesto teóricamente, y que encontró parcialmente apoyo en García-Ros et al. (2015), sí ajusta bien a los datos. Por otro lado, el modelo de un factor, mucho más parsimonioso, también presenta un buen ajuste. A favor del modelo de un factor se encuentra su parsimonia, pero sin embargo no es capaz de distinguir las obvias diferencias que se encuentran entre las dimensiones a priori en relación con otros constructos de su red nomológica. A favor del modelo de tres factores se encuentra que sus dimensiones claramente presentan validez discriminante, que la capacidad de generalización se ve incrementada con respecto a la del modelo de un factor (es como se está empleando la escala en investigación), pero las correlaciones excesivamente altas entre estos factores hacen difícil negar la idea de que tienen un constructo general a la base, cuanto menos.

Por tanto, y en vista de los resultados obtenidos, hemos decidido tentativamente retener el modelo de tres factores como el que mejor representa los datos. Esta decisión se tomó teniendo en cuenta los índices de ajuste y la comparación de las correlaciones de los modelos de un factor y de tres factores con su red nomológica. A pesar de que los índices de ajuste para ambos modelos son buenos, en la tabla de correlaciones se puede ver como las correlaciones con la red nomológica del modelo de un factor son muy semejantes a las del primer factor del modelo de tres factores (gestión del aula). Esto indicaría que el segundo y el tercer factor del modelo de tres factores tienen una explicación idiosincrásica de la autoeficacia docente, y por tanto cabe tenerlos en cuenta. Esta es una forma de valorar la validez discriminante de la solución de tres factores con respecto a variables que puedan ser de interés para el investigador. Si los factores que se asumen con base en las respuestas de los profesores presentan correlaciones diferentes con otros constructos, es que pueden añadir algo específico a la explicación en el entorno educativo, lo que apoyaría la utilidad de la estructura más compleja. Estos resultados están en línea con los resultados hallados en el estudio original de la escala (Brouwers \& Tomic, 2001), así como con los resultados obtenidos por García-Ros et al. (2015), quienes también defienden la estructura de tres factores. En el caso de la validación original, sin embargo, el ajuste no era plenamente satisfactorio de acuerdo a los criterios habituales. La validación en castellano de la esca- 
la sí presentó un buen ajuste. Sin embargo, este buen ajuste solamente se produjo tras liberar cinco covarianzas de error, lo que no es deseable en el modelado estructural salvo que esté bien justificado teóricamente (Bollen, 1989) pues supone declarar que un residual (varianza no explicada) correlaciona con otro residual. Adicionalmente, el método de estimación empleado por ambos trabajos está superado por mejores estimaciones que corrijan la falta de normalidad y continuidad de las variables. Pese a ello, nuestros resultados se encuentran en la línea de los ya encontrados, aunque generan dudas sobre la validez discriminante de las dimensiones propuestas.

La existencia de medidas de autoeficacia específicas en la población docente es importante porque el sentimiento de autoeficacia de los docentes para motivar y promover el aprendizaje afecta al éxito académico que sus estudiantes son capaces de alcanzar (Bandura, 1993). Pudiendo medir los niveles de autoeficacia docente de una población específica se podrán desarrollar, en caso de que sea necesario, políticas e intervenciones encaminadas a fomentar el sentimiento de autoeficacia en los profesores, a fin de lograr un mayor bienestar para estos y mayores niveles de éxito académico en la población. Además del éxito académico, la autoeficacia docente se relaciona con una serie de variables en los estudiantes: motivación (Bergman et al., 1977), autoestima y actitudes prosociales (Borton, 1991; Cheung \& Cheng, 1997). La autoeficacia docente se ha visto asociada también a una serie de variables organizacionales propias de los profesores: adopción de técnicas innovadoras (Fuchs, Fuchs, \& Bishop, 1992), compromiso profesional (Coladarci, 1992), absentismo (Imants \& Van Zoelen, 1995), estrés y burnout (Bliss \& Finneran, 1991). Según Velázquez (2012), la autoeficacia también influye positivamente en la competencia, hecho que resulta relevante si tenemos en cuenta el papel que juega la competencia en la labor de un maestro. Es por tanto importante tener a nuestra disposición herramientas adecuadas para medir la autoeficacia de los docentes, a fin de poder tener un control sobre la misma, pues esta influirá en una serie de factores cuyas consecuencias tienen un impacto directo sobre la calidad de la enseñanza.

En lo referente a potenciar el sentimiento de autoeficacia de los docentes, no deben olvidarse una serie de factores ajenos a ellos y que también influirán en el desarrollo de este sentimiento de autoeficacia: el nivel de rendimiento de los estudiantes, sus edades, el nivel de preparación del docente y el tamaño de la clase. Por tanto, una forma de potenciar la autoeficacia del docente será proporcionarle el medio y las herramientas necesarias. Si bien es cierto que variables tales como la edad de los estudiantes o el nivel del rendimiento de los mismos no pueden controlarse, otras como son el tamaño de la clase o el nivel de preparación del profesorado sí pueden controlarse. Por tanto, habrá que proporcionar al profesorado la preparación necesaria para su labor así como reducir, en la medida de lo posible, el tamaño de la clase donde el docente obra. En este aspecto, la posibilidad de tener una herramienta para medir el impacto que puedan tener estos cambios sin duda resulta de enorme utilidad, y de ahí deriva la necesidad así como la importancia de disponer de un instrumento que pueda medir la autoeficacia docente con certeza.

Con todo, resulta de vital importancia el estudio de la autoeficacia docente, así como su diferenciación en autoeficacia docente personal y general. La escala validada en la presente investigación contribuye al estudio de la autoeficacia docente personal. Las fortalezas de este estudio son: que constituye una contribución al cuerpo de conocimiento sobre el funcionamiento de la escala, que la muestra destaca por su naturaleza aleatoria y representativa, y que es la primera validación 
de esta escala realizada en una muestra obtenida en la República Dominicana. Las limitaciones y, por tanto, las posibles mejoras serían: conseguir una muestra representativa, no sólo de docentes de distritos educativos de Santo Domingo, sino de la República Dominicana y, para valorar posibles invarianzas, sería conveniente recoger un mayor número de varones en la muestra.

\section{Referencias}

Ashton, P. T., Olejnik, S., Crocker, L., \& McAuliffe, M. (1982). Measurement problems in the study of teachers' sense of efficacy. Annual Meeting of the American Educational Research Association. New York.

Ashton, P., Buhr, D., \& Crocker, L. (1984). Teachers' sense of efficacy: A self- or norm-referenced construct. Florida Journal of Educational Research, 26(1), 2941. Recuperado de https://eric.ed.gov/?id=ED323221

Bandura, A. (1990). Multidimensional scales of perceived academic efficacy. Stanford, CA: Stanford University.

Bandura, A. (1993). Perceived self-efficacy in cognitive development and functioning. Educational Psychologist, 28(2), 117-148. doi: 10.1207/ s15326985ep2802_3

Bandura, A. (1997). Self-efficacy: The exercise of control. New York: Freeman.

Bandura, A. (2000). Cultivate self-efficacy for personal and organizational effectiveness. En E. A. Locke (Ed.), Handbook of Principles of Organization Behavior (pp. 120-136). Oxford: Blackwell.

Bergman, P., McLaughlin, M., Bass, M., Pauly, E., \& Zellman, G. (1977). Federal programs supporting educational change: Vol. VII. Factors affecting implementation and continuation. Santa Monica, CA: RAND.

Bliss, J., \& Finneran, R. (1991). Effects of school climate and teacher efficacy on teacher stress. Annual
Meeting of the American Educational Research Association. Chicago.

Bollen, K. A. (1989). Structural Equation Modeling with Latent Variables. Mahwah, NJ: LEA.

Borton, W. M. (1991). Empowering teachers and students in a restructuring school: A teacher efficacy interaction model and the effect on reading outcomes. Annual Meeting of the American Educational Research Association. Chicago.

Brouwers, A. (2000). Teacher burnout and self-efficacy: An interpersonal approach. Heerlen, Netherlands: Open University.

Brouwers, A., \& Tomic, W. (2001). The factorial validity of scores on the teacher interpersonal self-efficacy scale. Educational and Psychological Measurement, 61(3), 433-445. doi: 10.1177/00131640121971301

Brouwers, A., Tomic, W., \& Stijnen, S. (2002). A confirmatory factor analysis of scores on the teacher efficacy scale. Swiss Journal of Psychology, 61(4), 211-219. doi: 10.1024/1421-0185.61.4.211

Chacón, C. (2006). Las creencias de autoeficacia: Un aporte para la formación del docente de inglés. Acción Pedagógica, 15(1), 44-54. Recuperado de http:// www.saber.ula.ve/handle/123456789/17262

Cheung, W. M., \& Cheng, Y. C. (1997). A multi-level analysis of teachers' self-belief and behavior, and students' educational outcomes. Annual Meeting of the American Educational Research Association. Chicago.

Coladarci, T. (1992). Teachers' sense of efficacy and commitment to teaching. Journal of Experimental Education, 60(4), 323-337. doi: 10.1080/00220973.1992.9943869

Emmer, E. T., \& Hickman, J. (1991). Teacher efficacy in classroom management and discipline. Educational and Psychological Measurement, 51(3), 755-765. doi: 10.1177/0013164491513027

Fernández, B. (2012). Un análisis multidimensional del síndrome de burnout en profesorado de conservatorios y enseñanza secundaria. (Tesis de Doctorado). Universidad de Valencia, España.

Fernández, J. (1987). Escala de Motivaciones Psicosociales. 
Madrid: Tea.

Finney, S. J., \& DiStefano, C. (2006). Non-normal and categorical data in structural equation modeling. En G. R. Hancock \& R. O. Mueller (Eds.), Structural equation modeling: A second course (pp. 269-314). Greenwich, CT: Information Age.

Fuchs, L. S., Fuchs, D., \& Bishop, N. (1992). Instructional adaptation for students at risk. Journal of Educational Research, 86(2), 70-84. doi: 10.1080/00220671.1992.9941143

García-Ros, R., Fuentes, M. C., \& Fernández, B. (2015). Teachers' interpersonal self-efficacy: Evaluation and predictive capacity of teacher burnout. Electronic Journal of Research in Educational Psychology, 13(3), 483-502. doi: 10.14204/ejrep.37.14105

Gibson, S., \& Dembo, M. H. (1984). Teacher efficacy: Aconstruct validation. Journal of Educational Psychology, 76(4), 569-582. doi: 10.1037/0022-0663.76.4.569

Gonzalo-Delgado, M., \& León del Barco, B. (1999). La promoción de la autoeficacia en el docente universitario. Revista Electrónica Interuniversitaria de Formación del Profesorado, 2(1), 79-90. Recuperado de https:// dialnet.unirioja.es/servlet/articulo?codigo=2794996

Guskey, T. R. (1981). Measurement of responsibility teachers assume for academic successes and failures in the classroom. Journal of Teacher Education, 32(3), 44-51. doi: 10.1177/002248718103200310

Henson, R. K., Kogan, L. R., \& Vacha-Haase, T. (2001). A reliability generalization study of the Teacher Efficacy Scale and related instruments. Educational and Psychological Measurement, 61(3), 404-420. doi: 10.1177/00131640121971284

Hu, L., \& Bentler, P. M. (1999). Cut-off criteria for fit indexes in covariance structure analysis: Conventional criteria versus new alternatives. Structural Equation Modeling, 6(1), 1-55. doi: 10.1080/10705519909540118

Imants, J., \& Van Zoelen, A. (1995). Teachers' sickness absence in primary schools, school climate and teachers' sense of efficacy. School Organization, 15(1), 77-86. doi: 10.1080/0260136950150109

McAdams, D. P., \& St. Aubin, E. (1992). A theory of gene- rativity and its assessment through self-report, behavioral acts, and narrative themes in autobiography. Journal of Personality and Social Psychology, 62(6), 1003-1015. doi: 10.1037/0022-3514.62.6.1003

Muthén, L. K., \& Muthén, B. O. (1998-2012). Mplus User's Guide ( $7^{\mathrm{a}}$ ed.). Los Angeles, CA: Muthén \& Muthén,

Prieto, L. (2007). Autoeficacia del profesor universitario. Eficacia percibida y práctica docente. Madrid: Narcea.

Riggs, I., \& Enochs, L. (1990). Toward the development of an elementary teacher's science teaching efficacy belief instrument. Science Education, 74(6), 625-638. doi: 10.1002/sce.3730740605

Rose, J. S., \& Medway, F. J. (1981). Measurement of teachers' beliefs in their control over student outcome. Journal of Educational Research, 74(3), 185-190. doi: 10.1080/00220671.1981.10885308

Tanaka, J. S. (1993). Multifaceted conceptions of fit in structural equation models. En K. A. Bollen \& J. S. Long (Eds.), Testing Structural Equation Models (pp. 10-39). Newbury Park, CA: Sage.

Velásquez-Fernández, A. (2012). Revisión histórico-conceptual del concepto de autoeficacia. Revista Pequén, 2(1), 148-160. Recuperado de http://revistas.ubiobio. cl/index.php/RP/article/view/1838 


\section{ANEXO I \\ Ítems de la escala Teacher Interpersonal Self-Efficacy Scale.}

\begin{tabular}{|c|c|}
\hline & Si un alumno interrumpe la clase, soy capaz de reconducirlo rápidamente. \\
\hline 2 & Soy capaz de acercarme a los directivos si quiero hablar de problemas con el trabajo. \\
\hline 3 & Confío en que, si es necesario, puedo pedir consejo a mis compañeros. \\
\hline 4 & Son muy pocos los alumnos que no puedo soportar. \\
\hline 5 & Puedo sobrellevar los estudiantes más complicados. \\
\hline 6 & Cuando es necesario, soy capaz de sacar a colación problemas con los directivos. \\
\hline 7 & Siempre puedo encontrar compañeros con los que puedo hablar de problemas en el trabajo. \\
\hline 8 & Puedo tomar medidas adecuadas para mantener actividades funcionando de manera adecuada. \\
\hline 9 & Puedo decirles a los alumnos que me tomo muy en serio conseguir un trato apropiado. \\
\hline 10 & No siempre soy capaz de ejecutar varias actividades a la vez. \\
\hline 11 & Puedo manejar mi clase bastante bien. \\
\hline 12 & Confío en que, si es necesario, puedo conseguir que los directivos me ayuden. \\
\hline 13 & Puedo mantener a los alumnos desafiantes implicados en la clase. \\
\hline 14 & Soy siempre capaz de expresar de forma clara mis expectativas a los alumnos. \\
\hline 15 & Soy capaz de responder de manera adecuada a estudiantes desafiantes. \\
\hline 16 & Cuando es necesario soy capaz de conseguir que los directivos me apoyen. \\
\hline 17 & Puedo evitar que unos pocos alumnos desafiantes me arruinen una clase entera. \\
\hline 18 & Si los alumnos paran de trabajar puedo devolverles a la tarea. \\
\hline 19 & Confío en que si es necesario puedo pedir consejo a los directivos. \\
\hline 20 & Si me enfrento a un problema en el que mis compañeros me pueden ayudar, soy capaz de acercarme a ellos para ello. \\
\hline 21 & Cuando es necesario soy capaz de pedir ayuda a un compañero. \\
\hline 22 & Sé qué reglas son las apropiadas para mis alumnos. \\
\hline 23 & Soy capaz de acercarme a mis compañeros si quiero comentar problemas sobre el trabajo. \\
\hline 24 & Soy capaz de empezar el curso de manera que los alumnos aprenderán a comportarse bien. \\
\hline
\end{tabular}

